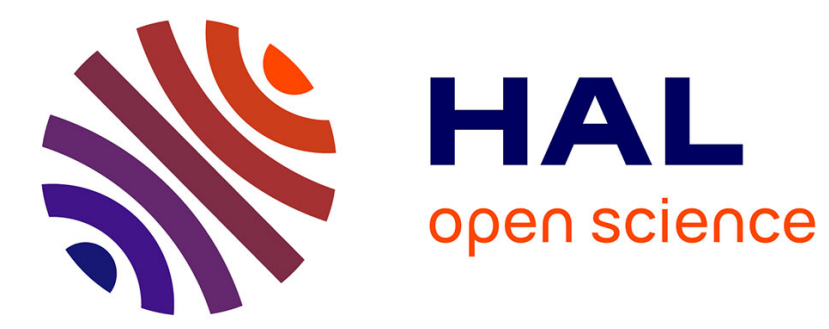

\title{
MODÉLISATION DE MICROPHONES SUB-MINIATURES
}

\author{
G. Plantier, M. Bruneau
}

\section{To cite this version:}

G. Plantier, M. Bruneau. MODÉLISATION DE MICROPHONES SUB-MINIATURES. Journal de Physique Colloques, 1990, 51 (C2), pp.C2-555-C2-558. 10.1051/jphyscol:19902130 . jpa-00230425

\section{HAL Id: jpa-00230425 https://hal.science/jpa-00230425}

Submitted on 1 Jan 1990

HAL is a multi-disciplinary open access archive for the deposit and dissemination of scientific research documents, whether they are published or not. The documents may come from teaching and research institutions in France or abroad, or from public or private research centers.
L'archive ouverte pluridisciplinaire HAL, est destinée au dépôt et à la diffusion de documents scientifiques de niveau recherche, publiés ou non, émanant des établissements d'enseignement et de recherche français ou étrangers, des laboratoires publics ou privés. 
COLLOQUE DE PHYSIQUE

Colloque C2, supplément au $n^{\circ} 2$, Tome 51, Février 1990

ler Congrès Français d'Acoustique 1990

\title{
MODÉLISATION DE MICROPHONES SUB-MINIATURES
}

\section{G. PLANTIER(1) et M. BRUNEAU}

Laboratoire d'Acoustique, CNRS URA 1101, Université du Maine, BP. 535, F-72017 Le Mans Cedex, France

\begin{abstract}
Résumé - La tendance générale à la miniaturisation et l'évolution des techniques de micro-usinage sont à l'origine de la réalisation de capteurs silicium électro-statiques ou électrets miniatures. Certaines dimensions intérieures de ces capteurs étant de l'ordre de l'épalsseur des couches limites, leur modélisation précise nécessite l'usage des équations de propagation en fluide viscothermique. Dans ce cadre, un calcul de réponse de membrane séparée d'une électrode par un film d'air très mince a été effectué et les modifications à apporter aux résultats disponibles jusqu'à présent, qui admettent l'hypothèse isotherme, sont présentées.
\end{abstract}

Abstract - The application of semiconductor fabrication techniques to the production of minlature électro-static and electret sensors has necessitated the theoretical modelling of mechanical behaviour at very small scale. Since the internal dimensions of such silicon transducers are comparable with that of the boundary layer we have produced an improved theory to describe the transducer behaviour which uses the equations of propagation for visco-thermal fluids.

Using this theory, which does not assume the processes to be isothermal, we have calcultated the acoustic response of a stretched membrane separated from a rigid electrode by a very thin air film, and we show a comparison of our results with those available in the literature.

\section{1 - INTRODUCTION}

Les développements récents de systèmes acoustiques miniaturisés, conjugués à l'évolution des techniques de micro-usinage, sont à l'origine d'études de capteurs microphoniques miniatures électrostatiques réalisés sur support sllicium $/ 1,2,3 \%$. La modélisation précise de tels capteurs requiert une description des mouvements de gaz dans les cavités internes qui soit adaptée aux exigences liées aux très faibles dimensions de ces cavités. Dans ce cadre, nous proposons ici le calcul de la réponse acoustique d'une membrane tendue, fixée de façon rigide sur son pourtour, séparée d'une électrode rigide par un film de fluide très mince, dont l'épaisseur est de l'ordre de grandeur de celle des couches limites visqueuses et thermiques aux parois (1 à $10 \mu \mathrm{m})$, ce film fluide débouchant à sa périphérie sur un réservoir de volume beaucoup plus grand (Fig. 1 ).

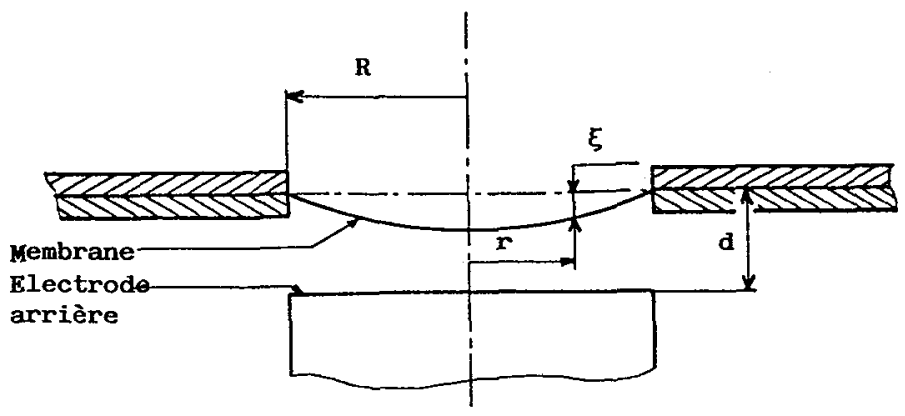

Fig. 1 - Vue en coupe d'une membrane circulaire tendue et de I'électrode associée.

(1) Supported by the Badin-Crouzet Company (Convention CIFRE) 
L'objectif du travall est plus précisément d'améliorer les théories en usage, qui s'avèrent insuffisantes dans les estimations envisagées ici, en refusant pour l'essentiel l'hypothèse de transformation isotherme dans le flim fluide pour y substituer une théorie qui prend en compte les transferts thermiques plus conformes à la réalité, conservant par allleurs dans les équations les facteurs prenant notamment en compte les effets de viscosité.

\section{2 - IA THEORIE FONDAMENTALE}

La formulation du problème acoustique repose sur la théorie classique de la propagation en fluide visco-thermique $/ 4 /$, admettant, sur les frontières rigides, une vitesse particulaire totale nulle et une température instantanée constante, et sur la membrane la continuité de la vitesse instantanée (et bien sûr encore la constance de la température instantanée). Cette formulation, qui prend en compte les effets liés aux modes entropiques, étant une extension de celle actuellement disponible dans la littérature, proposée par D.H. Robey $/ 5 /$, qui admet l'hypothèse isotherme, les conditions retenues $1 \mathrm{cl}$ a l'interface entre le film d'air et le réservolr sont celles de cet auteur (impédance du réservoir nulle), à fins de comparaisons significatives ( 1 'extension à des conditions plus réalistes à cet endroit ne présente aucune difficulté de principe).

En terme (notamment) de vitesse particulaire, les équations du problème admettent trois types de solutions (4): acoustique, entropique et tourbilionnaire. La première solution obéit à une équation de propagation, les deux autres à des équations de diffusion traduisant respectivement les effets de conduction thermique et de viscosité. Les longueurs de diffusion des vitesses entropiques et tourblllonnaires sont très inférieures aux longueurs d'ondes, dans les domaines de fréquences pour lesquels ces capteurs sont conçus, mais restent de l'ordre de grandeurs de l'intervalle qui sépare la membrane de l'électrode arrière (quelques $\mu \mathrm{m}$ ). Les mouvements de fluide dans cet espace sont ceux de couches limites dans lesquelles les trois "composantes" de la vitesse particulaire totale interagissent fortement, par suite des conditions aux frontières imposées à la vitesse particulaire totale et à la température instantanée totale.

\section{3 - RESULTATS EXPERIMENTAUX}

Le problème a été résólu dans le cas d'une membrane et d'une électrode arrière circulaires, dont les caractéristiques géonétriques sont exactement celles retenues pour l'auteur déjà cité $/ 5 \%$ à fins de comparaison des résultats. La valeur moyenne du déplacement de la membrane et son impédance ont été calculées.

Dans un développement sur une base de fonctions propres, le nombre de modes retenus est celui qui nous a permis d'obtenir les résultats de H. Robey lorsque I' on admet l'hypothèse du gaz parfaitement conducteur de la chaleur. c'est-à-dire lorsque l'on admet que le processus est isotherme (et lorsque I'on néglige l'influence direct de la viscosité sur le mouvement acoustique, sans négliger pour autant le mouvement tourbillonnaire - mais ce facteur est de peu d'importance).

La figure (2) présente, en unité arbitraire, le déplacement moyen de la membrane $\langle\xi\rangle$ divisé par la pression acoustique incidente supposée uniforme. La courbe en pointillés reproduit le résultat de la référence $/ 5 /$, qui admet l'hypothèse isotherme, et le trait continu correspond à nos résultats qui prennent en compte les effets de conduction thermique, ce qui signifie d'une certaine manière que le comportement du gaz se situe entre celui de 
1' Isotherme et celul de l'adiabatique aux fréquences élevées (Icl entre $10^{2}$ et $10^{4} \mathrm{~Hz}$ ). La figure 3, présentée comme la flgure 2, donne les partles réelles $(\mathcal{H}$ ) et imaginalres $(\mathcal{J})$ de $l$ 'impédance de la membrane, rapport de la pression acoustique incidente a la vitesse moyenne de la membrane. En plus des écarts attendus dans les hautes fréquences, on constate une différence entre les partles réelles en basses fréquences, alors qu' une telle différence n'apparait pas sur la figure 2 . Cet écart en basses fréquences vient de ce que la partle réelle ne dépend là que de termes petits d'ordre supérleur dans les calculs, qul sont sensibles a la description cholsi, pour décrire la diffusion thermique, et qu'elle est de ce falt très falble en comparaison de la partie imaginaire.

La "transitlon" mentionnée aux fréquences élevées entre le comportement de l'adiabatique et celul de l'isotherme peut avoir une influence sur le comportement des transducteurs subminlatures dans les domaines de fréquences en usage dans certaínes applications ; c'est là que la formulation proposée prend tout son sens.

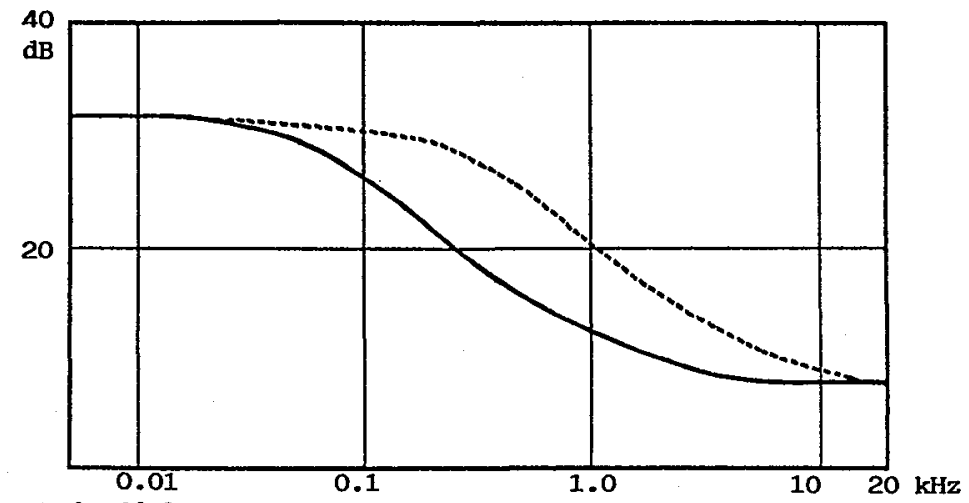

Fig. 2 - Rapport du déplacenent moyen $\zeta$ de la membrane et de la pression incidente uniforme acoustique $p_{i}$, en unité arbitraire, en fonction de la fréquence. La ligne continue correspond au résultat obtenu au terme de notre calcul (qui tient compte des effets de conduction thermique), la ligne en pointillés reproduit les résultats de la référence /5/ (qui admettent que les phénomènes sont isothermes).

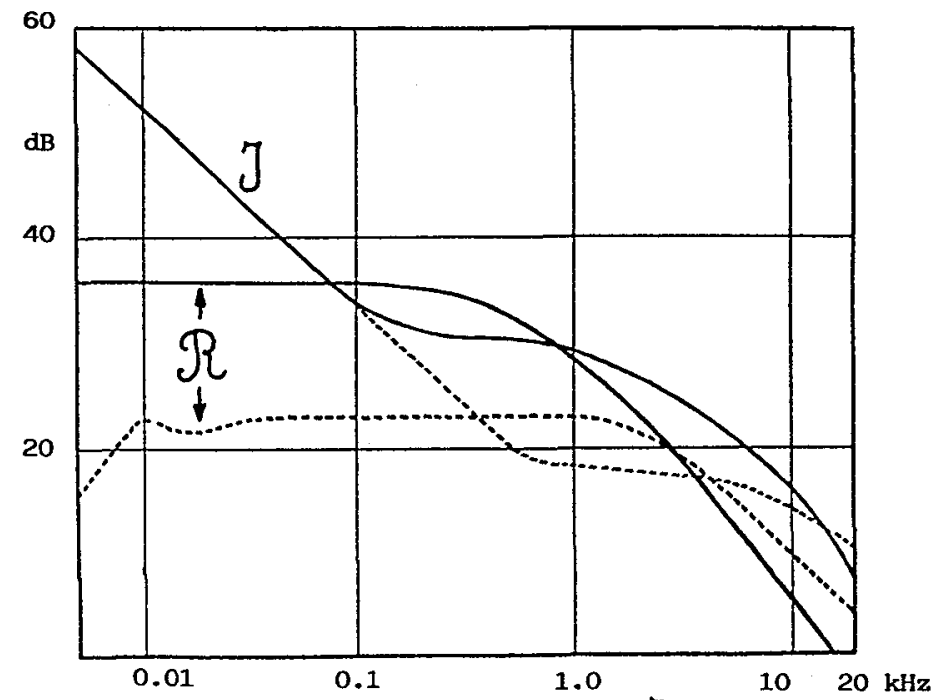

Fig. 3 - Partie réelle $(\mathcal{L})$ et partie imaginaire $(J)$ de l'impédance de membrane, rapport de la pression acoustique incidente et de la vitesse moyenne associée. Les lignnes continues correspondent à nos résultats, les lignes en pointillés reproduisent ceux de la référence $/ 5 /$. 


\section{REFERENCES}

/1/ Poteat, T.L. and Bush-Vishniac, Silicon Micromechanics : New Technology for sensors, I.C. A. Toronto (1986).

/2/ Bush-Vishniac et al., U.S. Patent $\underline{4}$ 184, Dec 1985.

13/ Hohm Dietmar, Darmstadt - ISBN 3 - 18 - 146019 - 9 (1986).

/4/ Bruneau, M. et al., Wave Motion 11, (1989) 441-451.

/5/ Robey, D.H., J. Acoust. Soc. Am. VoI $26 \underline{5}$ (1954) 740-745. 